\title{
Time to switch laboratories
}

Do you want to work with a full-service dental laboratory that will put your work in safe hands with both dedicated NHS and private departments?

CosTech Dental Laboratory has been helping its dentists to 'create smiles to live for' since 1977 . With the market saturated by so many restorations and available technologies, CosTech says it will be your one point of contact.

Its experts can help you with case planning and trouble-shooting, as well as providing a base for knowledge and support.

CosTech offers a full range of products to suit every case a dentists sees. From crowns and bridges, to custom and stock implant systems, both you and your patients will benefit from outstanding precision, quality and reliability.

CosTech's digital department can also create the perfect restorations by using your digital scan files.

Why CosTech?

- Well established lab with 40 years of experience

- Full service laboratory

- Dedicated NHS and private departments

\section{The new industry standard}

Want a temporisation material that sets a new standard of excellence? Protemp 4 temporisation material from 3M Oral Care supplies a cutting-edge blend of scientific research and aesthetic excellence unlike any other.

Due to its high compressive, impact and tensile strength, ${ }^{1}$ Protemp 4 is well suited to demanding applications such as long-term temporisation. Furthermore, as it requires minimal preparation (no glaze or polish), ${ }^{2}$ it offers a fast solution every time.

Aesthetics matter and Protemp 4 has been created to offer a natural-looking solution. ${ }^{3}$ The material even contains fluorescence pigments that allow it to remain looking natural under challenging conditions such as UV light. ${ }^{4}$

For more information, call 0800626578 or visit www.3M. co.uk/Dental.

$3 \mathrm{M}$ and Protemp 4 are trademarks of the 3M Company.

1. 3M Oral Care internal data. High compressive, impact, tensile strength. Claim 3445 . 2009.

2. 3M Oral Care internal data. Minimal preparation. Claim 3627. 2008.

3. 3M Oral Care internal data. Natural looking aesthetics. Claim 3422. 2008.

4. 3M Oral Care internal data. Natural even in UV light. Claim 3423. 2008.

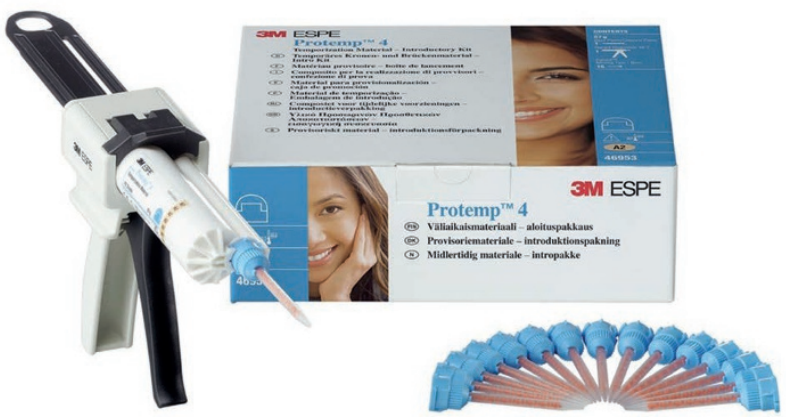

- Consistent and reliable

- Services whole of the UK and offers a delivery service in the South East

- Committed to quality, DAMAS registered, compliant since 2011.

For more information about CosTech Dental Laboratory, please visit www.costech.co.uk or call 01474320076.

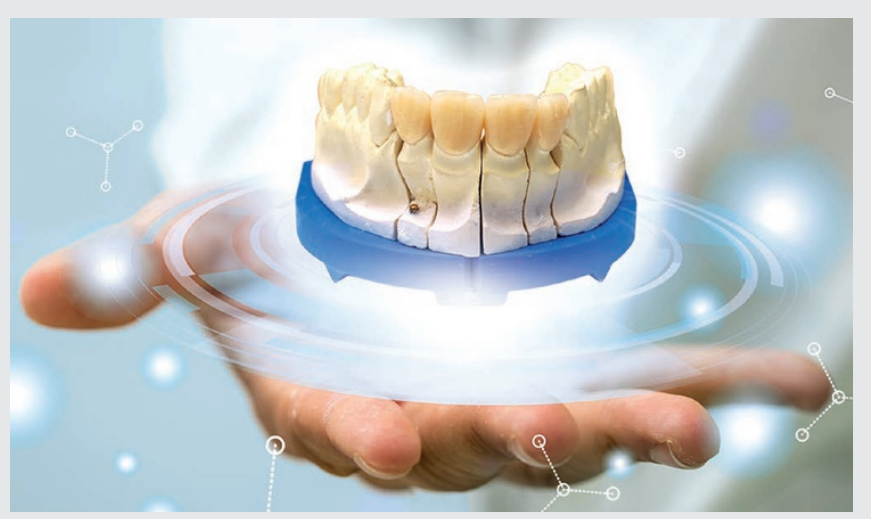

\section{Preventive dentistry through bio-technology}

A year after the launch of the CALCIVIS imaging system, Dr Chris Longbottom has given his views on the product that evolved from his research on bioluminescence.

The CALCIVIS imaging system is described as a 'first for dentistry' making it possible for practitioners to visualise active demineralisation on tooth surfaces.

'The CALCIVIS imaging system has been developed superbly and is precisely what we need to stimulate patients and clinicians,' said Longbottom.

'The images that can be created are exactly as I had originally hoped and the feedback that we have received from patients and practitioners is excellent. This validates everything that we have been working for years to achieve and I feel a mixture of pride, elation and excitement.'

The CALCIVIS imaging system is said to be the first bio-tech dental product in the world and Longbottom added: 'This technology provides a system that assists clinicians with caries diagnostic assessments at the very earliest stages of disease and aids communication substantially.

'Patients can relate to the images, which arouses all kinds of positives. It increases their awareness of their own oral health, helps them to understand the disease process and motivates them in terms of prevention.'

To find out more about CALCIVIS visit www.CALCIVIS.com or call 01316585152.

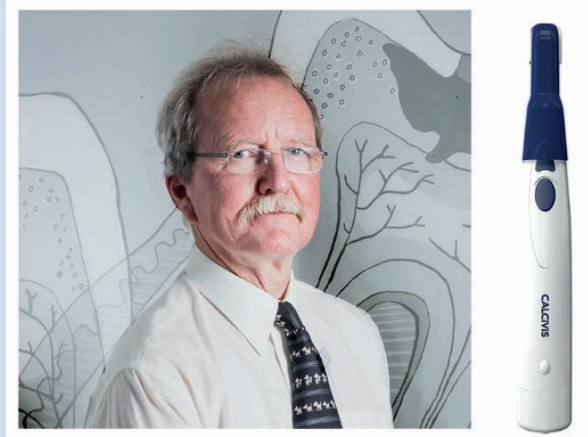

